\title{
Association of the PPAR $\gamma /$ PI3K/Akt pathway with the cardioprotective effects of tacrolimus in myocardial ischemic/reperfusion injury
}

\author{
XIUFEN LI, AISHAN BILALI, RUI QIAO, TUERXUN PAERHATI and YAN YANG
}

Cardiac Care Unit, The Traditional Hospital Affiliated to Xinjiang Medical University, Ürümqi, Xinjiang 830001, P.R. China

Received May 18, 2017; Accepted November 2, 2017

DOI: $10.3892 / \mathrm{mmr} .2018 .8649$

\begin{abstract}
Myocardial ischemia/reperfusion injury (MIRI) induces severe arrhythmias and has a high risk of mortality. The aim of the present study was to investigate the effect of tacrolimus on arrhythmias, cardiac function, oxidative stress and myocardium apoptosis induced by MIRI, and to elucidate the underlying mechanism. The effects of MIRI and tacrolimus on arrhythmias, cardiac function parameters, myocardial oxidative stress and apoptosis were investigated in a rat model of MIRI. The phosphorylation of peroxisome proliferator-activated receptor $\gamma$ (PPAR $\gamma)$ and protein kinase $\mathrm{B}$ (Akt) was investigated via western blotting. After rats were treated with inhibitors of PPAR $\gamma /$ phosphoinositide 3-kinase (PI3K)/Akt, cardiac function parameters were measured. The results demonstrated that the MIRI procedure induced arrhythmias and significant impairment of cardiac function, oxidative stress and apoptosis in cardiomyocytes $(\mathrm{P}<0.05)$. Tacrolimus significantly alleviated the arrhythmias and impairment of cardiac function and inhibited the oxidative stress and apoptosis in cardiomyocytes $(\mathrm{P}<0.05)$. The phosphorylation of PPAR $\gamma$ and Akt was significantly activated by tacrolimus, whereas inhibitors of PPAR $\gamma / \mathrm{PI} 3 \mathrm{~K} / \mathrm{Akt}$ significantly abolished the effects of tacrolimus $(\mathrm{P}<0.05)$. Together, these results suggest that tacrolimus may protect rats from MIRI through activation of the PPAR $\gamma / \mathrm{PI} 3 \mathrm{~K} / \mathrm{Akt}$ pathway.
\end{abstract}

\section{Introduction}

Primary percutaneous coronary intervention (PCI) is an effective treatment method for patients with acute myocardial ischemia (1). As the duration of ischemia is a critical factor in infarct size and patient prognosis, the time interval between

Correspondence to: Dr Tuerxun Paerhati, Cardiac Care Unit, The Traditional Hospital Affiliated to Xinjiang Medical University, 116 Huanghe Road, Ürümqi, Xinjiang 830001, P.R. China E-mail: tuerxun_xj@sina.com

Key words: tacrolimus, myocardium ischemic/reperfusion injury, peroxisome proliferator-activated receptor $\gamma$, protein kinase B, phosphoinositide 3-kinase chest pain onset and PCI treatment should be minimized (1). It has previously been demonstrated that rapid restoration of blood flow to the ischemia area via PCI is effective in increasing the survival of patients with acute myocardial ischemia (2). Conversely, there is evidence suggesting that PCI increases the risk of heart failure in patients with acute myocardial ischemia, as it leads to myocardial injury and cardiomyocyte death, which is also known as myocardial ischemia/reperfusion (I/R) injury (MIRI) (3). The pathogenesis of MIRI includes marked accumulation of reactive oxygen species (ROS) and cardiomyocyte apoptosis (4), which may subsequently induce cardiomyocyte damage, myocardial function impairment and lethal arrhythmias (5). ROS are generated from injured myocytes, endothelial cells and neutrophils in the ischemic zone, and are further activated by reperfusion (6). The over-production of ROS facilitates membrane damage and calcium overload, which leads to cell membrane lipid peroxidation, protein denaturation and DNA damage (7). Apoptosis is associated with infarct size and left ventricular remodeling during MIRI (8). It is induced by activation of sarcolemmal receptors via an extrinsic mechanism, or by mitochondrial release of cytochrome $c$ via an intrinsic mechanism (9). The mitochondrial permeability transition pore (MPTP) is an essential component of cell apoptosis (10). MPTP opens as a result of increased concentrations of calcium and ROS, or a decreased inner mitochondrial membrane potential, all of which are present in MIRI $(11,12)$. To improve the outcomes of patients with acute myocardial ischemia after receiving PCI or other reperfusion therapies, a prevention strategy against MIRI needs to be developed.

Tacrolimus (also known as FK506) is a macrolide antibiotic compound that was discovered in 1984. It is a metabolite of the fungus Streptomyces tsukubaensis (13). As a calcineurin inhibitor, it has typically been used as an immunosuppressive agent in organ transplantations and autoimmune diseases since the mid-1980s $(14,15)$. It is able to bind to FK506 binding protein and inhibit calcineurin phosphatase, which in turn inhibits calcium-dependent events, including gene transcription of interleukins, nitric oxide synthase activation and apoptosis (13). Previous studies have demonstrated that tacrolimus alleviates I/R injury in various organs, including the ovary, liver and brain $(16,17)$. It has also been revealed that tacrolimus may exert anti-oxidative and anti-apoptotic 
effects $(18,19)$. However, the effects of tacrolimus on MIRI and the exact mechanism are yet to be elucidated.

As a type II nuclear receptor, peroxisome proliferator-activated receptor $\gamma$ (PPAR $\gamma)$ is associated with the pathology of a number of diseases. It is able to reduce ROS stress and apoptosis during I/R injury (20). There is also an association between PPAR $\gamma$ and tacrolimus. FK506 binding protein 51 (FKBP51) has previously been identified as a chaperone to PPAR $\gamma$, and FKBP51 over-expression was demonstrated to increase PPAR $\gamma$ activity, in which Akt phosphorylation was associated (21). Therefore, in the present study, the effects of tacrolimus on MIRI-induced arrhythmias and heart function were evaluated, and the oxidative products and apoptosis proteins in the myocardium were measured. The phosphorylation of PPAR $\gamma$ and protein kinase B (Akt) was subsequently measured by western blotting to observe the activation of the PPAR $\gamma /$ phosphoinositide 3-kinase (PI3K)/Akt pathway. Finally, via the use of PPAR $\gamma / \mathrm{PI} 3 \mathrm{~K} / \mathrm{Akt}$ pathway inhibitors, heart function parameters were examined to investigate the role of the PPAR $\gamma / \mathrm{PI} 3 \mathrm{~K} / \mathrm{Akt}$ pathway.

\section{Materials and methods}

Animals. Adult male Wistar rats $(n=140 ;$ mean weight, $250 \pm 20 \mathrm{~g}$; age, 45-55 days) were purchased from the Animal Center of Xinjiang Medical University (Ürümqi, China) and housed in a climate-controlled room (temperature, $25 \pm 2{ }^{\circ} \mathrm{C}$; humidity, 50-60\%) in the Animal Center of Xinjiang Medical University with a 12-h light-dark cycle and ad libitum access to food and water. In the part A experiment, rats were randomly assigned to four groups, with 8 or 12 rats per group. In the experiment Part A, 88 rats were assigned to sham+vehicle, sham+tacrolimus, I/R+vehicle and $\mathrm{I} / \mathrm{R}+$ tacrolimus groups $(\mathrm{n}=22)$. For biomedical assays, there were 10 rats per group; for cardiac function measurements, there were 12 rats per group. In the experiment Part B, 48 rats were assigned to $\mathrm{I} / \mathrm{R}+$ tacrolimus+vehicle, I/R+tacrolimus+GW9662, I/R+tacrolimus+LY294002 and $\mathrm{I} / \mathrm{R}+$ tacrolimus+triciribine groups $(\mathrm{n}=12)$. All experiments were performed in accordance with the guidelines set by the National Institutes of Health (22) and approved by Animal Ethics Committee of Xinjiang Medical University. Tacrolimus (0.1 mg/kg; Sigma-Aldrich; Merck KGaA, Darmstadt, Germany) was intraperitoneally administered to rats in the treatment groups $30 \mathrm{~min}$ prior to MIRI procedure. Rats in the vehicle groups were administered with the same volume of saline. The PPAR $\gamma$ inhibitor GW9662, PI3K inhibitor LY294002 and Akt inhibitor triciribine were purchased from Sigma-Aldrich (Merck KGaA). GW9662, LY294002 or triciribine were administered intraperitoneally at $30 \mathrm{~min}$ prior to tacrolimus injection at dosages of $2 \mathrm{mg} / \mathrm{kg}, 100 \mathrm{mg} / \mathrm{kg}$ and $2 \mathrm{mg} / \mathrm{kg}$, respectively. Twelve rats were administered with each inhibitor. Twelve rats in the $\mathrm{I} / \mathrm{R}+$ tacrolimus+vehicle group received same volume of saline. The experimental groups and procedure are summarized in Fig. 1.

MIRI model. The MIRI procedure was performed according to the method recently detailed by Zhou et al (23). Briefly, the left coronary artery was exposed via surgical incision. A 3-0 silk braided suture was used to form a snare around the left coronary artery. Tightening and releasing this snare was performed via a Voss clip. Ischemia was maintained for $1 \mathrm{~h}$ by tightening the snare, which was then released for reperfusion. The duration of reperfusion was $1 \mathrm{~h}$. When reperfusion was completed, rats were returned to their cages for a 2-week recovery period. Buprenorphine hydrochloride (Sigma-Aldrich; Merck KGaA) was intramuscularly administered $(0.65 \mathrm{mg} / \mathrm{kg})$ to reduce postoperative pain. Sham rats underwent a similar surgical procedure wherein the coronary artery was not snared.

Cardiac function measurements. Evaluation of arrhythmia and mean blood pressure (MBP) was performed prior to and during the MIRI procedure according to protocols recently described by $\mathrm{Su}$ et al (24). Heart rate, ventricular tachycardia and fibrillation were recorded in rats of $\mathrm{I} / \mathrm{R}$ groups during $\mathrm{I} / \mathrm{R}$, or in the sham group rats at the corresponding time period via AcqKnowledge waveform analysis software (version 4.1; Biopac Systems, Inc., Goleta, CA, USA) based on diagnostic criteria from The Lambeth Conventions guidelines (25). Incidences and durations of ventricular tachycardia and fibrillation were counted from the ECG. Cardiac function measurements were performed as recently described by Sivasinprasasn et al (26) at 2 weeks following the MIRI procedure. Briefly, following exposure of the right carotid artery, a micro-tipped transducer catheter was inserted into the right carotid artery to the left ventricle (LV). The other end of the catheter was connected to a transducer control unit and a Grass model 7 polygraph (Natus Medical Incorporated, Pleasanton, CA). The LV end-diastolic pressure (LVEDP), LV systolic pressure (LVSP), maximal rates of rise in LV pressure (dP/dtmax), maximal rates of fall in LV pressure (- $\mathrm{dP} / \mathrm{dtmin})$ and stroke volume (SV) were recorded and the mean was calculated from 10 consecutive beats. Rats were monitored for survival/mortality for two weeks after the I/R procedure.

Assessment of malondialdehyde (MDA), protein carbonyl and 8-hydroxy-2-deoxyguanosine (8-OHdG). Immediately following the completion of reperfusion, myocardial tissue was harvested and homogenized at $4^{\circ} \mathrm{C}$ for $10 \mathrm{~min}$ in a potassium phosphate buffer solution $(50 \mathrm{mM}$; pH 7.5). Following centrifugation at $1,500 \mathrm{x} \mathrm{g}$ and $4^{\circ} \mathrm{C}$ for $10 \mathrm{~min}$, the supernatant was collected and placed on ice and measured for the levels of MDA, protein carbonyl and 8-OHdGaccording to previously described methods (27-29). Protein concentration was measured using a bicinchoninic acid protein assay kit (Sigma-Aldrich; Merck KGaA).

Western blotting. Western blotting was performed according to the protocols recently described by Musman et al (30). Cardiac tissues were homogenized in lysis buffer containing $50 \mathrm{mM}$ Tris- $\mathrm{HCl}$ (pH 7.4), 150 mM NaCl, $1 \%$ NP-40, 0.1\% SDS, 1 mM protease inhibitor cocktail and protein phosphatase inhibitor (Roche Applied Science, Madison, WI, USA) on ice for $10 \mathrm{~min}$ and the supernatant was collected following centrifugation at $12,000 \mathrm{x} \mathrm{g}$ at $4^{\circ} \mathrm{C}$ for $15 \mathrm{~min}$. Protein concentration was measured using a bicinchoninic acid protein assay kit (Sigma-Aldrich; Merck KGaA). A total of $40 \mu \mathrm{g}$ protein was loaded per lane and separated by $12 \%$ SDS-PAGE. The protein was then transferred to nitrocellulose membranes electrophoretically. Membranes 
A

\begin{tabular}{|l|}
\hline Sham+Vehicle \\
Sham+Tacrolimus \\
I/R+Vehicle \\
I/R+Tacrolimus \\
\hline
\end{tabular}

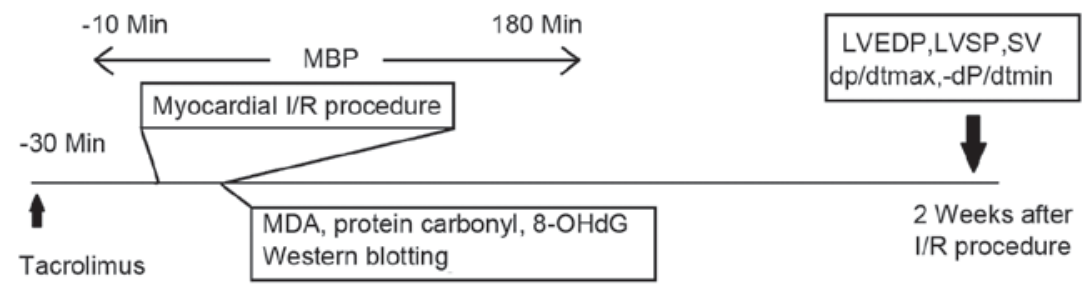

B
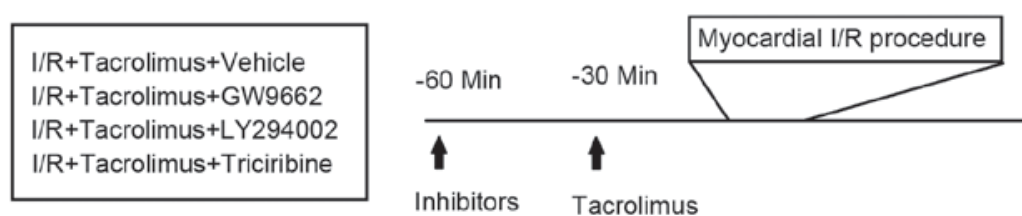

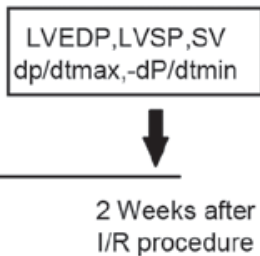

Figure 1. Experimental design and groups. The time of myocardial I/R procedure was considered as 0 min. (A) Protocols involving the sham and I/R groups. (B) Protocols involving the treatment groups within the I/R group. I/R, ischemia/reperfusion; MDA, malondialdehyde; 8-OHdG, 8-hydroxy-2-deoxyguanosine; LV, left ventricle; LVEDP, LV end-diastolic pressure; LVSP; LV systolic pressure; dP/dtmax, maximal rates of rise in LV pressure; -dP/dtmin: maximal rates of fall in LV pressure; SV, stroke volume.

were subsequently incubated with $5 \%$ non-fat milk at $25^{\circ} \mathrm{C}$ for $2 \mathrm{~h}$. Membranes were then incubated with primary antibodies for PPAR $\gamma$ (cat. no. sc-7273; 1:200; Santa Cruz Biotechnology, Inc. Dallas, TX, USA), phosphorylated (p)-PPAR $\gamma$ (Ser273; 1:800; New England Peptide, Inc., Gardner, MA, USA), Akt (cat. no. sc-5298; 1:1,000; Santa Cruz Biotechnology, Inc.), p-Akt (cat. no. sc-514032; 1:500; Santa Cruz Biotechnology, Inc.), Bax (cat. no. sc-493; 1:500; Santa Cruz Biotechnology, Inc.), caspase-3 (cat. no. sc-7148; 1:800; Santa Cruz Biotechnology, Inc.), cleaved caspase-3 (cat. no. sc-22171; 1:200; Santa Cruz Biotechnology, Inc.) and Bcl-2 (cat. no. sc-7382; 1:500; Santa Cruz Biotechnology, Inc.) at $4{ }^{\circ} \mathrm{C}$ overnight. Membranes were subsequently incubated with horseradish peroxidase-conjugated goat anti-rabbit IgG secondary antibody (cat. no. sc-2030; 1:300; Santa Cruz Biotechnology, Inc.) in tris-buffered saline/Tween-20 solution for $1 \mathrm{~h}$ at $25^{\circ} \mathrm{C}$. Blots were incubated with enhanced chemiluminescent detection solution (Pierce; Thermo Fisher Scientific, Inc., Waltham, MA, USA) and quantified by densitometry using Quantity One software 4.6.2 (version 4.6.2; Bio-Rad Laboratories, Inc., Hercules CA, USA).

Statistical analysis. Statistical analyses were performed using one-way analysis of variance followed by the Student-Newman-Keuls post hoc test, via SPSS 17.0 (SPSS, Inc., Chicago, IL, USA). Data are presented as the mean \pm the standard error of the mean. $\mathrm{P}<0.05$ was considered to indicate a statistically significant difference.

\section{Results}

Experimental design and groups. Fig. 1 details the experimental design and groups used in the present study. The time of myocardial I/R procedure was considered as $0 \mathrm{~min}$. Fig. $1 \mathrm{~A}$ details protocols involving the sham and I/R groups. Rats were divided into four groups: sham+vehicle, sham+tacrolimus, $\mathrm{I} / \mathrm{R}+\mathrm{vehicle}$ and $\mathrm{I} / \mathrm{R}+$ tacrolimus, and arrhythmias, cardiac function, MBP, oxidative products, apoptotic proteins, PPAR $\gamma /$ PI3K/Akt expression and phosphorylation were examined. Fig. 1B details protocols involving the different treatment groups within the I/R group. Rats were divided into four groups: I/R+tacrolimus+vehicle, I/R+tacrolimus+GW9662, I/R+tacrolimus+LY294002 and I/R+tacrolimus+triciribine, and cardiac function was examined.

Effect of tacrolimus on myocardial ischemia-reperfusion induced arrhythmias. Table I details the effect of tacrolimus on myocardial I/R-induced arrhythmias. No ventricular tachycardia or ventricular fibrillation was observed in the sham groups. In the I/R groups, there was no significant difference between the vehicle and tacrolimus groups in the incidence of ventricular tachycardia, but a significant decrease was observed in the tacrolimus group compared with the vehicle group in the duration of ventricular tachycardia, and the incidence and duration of ventricular fibrillation $(\mathrm{P}<0.05)$. The mortality rate of the tacrolimus group was also significantly lower than the vehicle group $(\mathrm{P}<0.05)$.

Cardiac function changes by myocardial ischemia-reperfusion and tacrolimus. Table II details the changes in cardiac function parameters [heart rate, LVESP, LVEDP, dP/dtmax, -dP/dtmin and SV/body weight (BW)] induced by myocardial $\mathrm{I} / \mathrm{R}$ and tacrolimus. In the sham group, there was no significant difference in any parameters between the vehicle and tacrolimus groups. In the $\mathrm{I} / \mathrm{R}$ groups there was no significant difference between the vehicle and tacrolimus groups in heart rate, but the LVESP, $\mathrm{dP} / \mathrm{dtmax}$ and SV/BW were significantly decreased, whereas the LVEDP and - $\mathrm{dP} / \mathrm{dtmin}$ were significantly increased in the vehicle vs. the sham group $(\mathrm{P}<0.05)$. Compared with the vehicle sham group, the heart rate, LVESP, $\mathrm{dP} / \mathrm{dtmax}$ and SV/BW were significantly decreased, whereas the LVEDP and - dP/dtmin were significantly increased in the $\mathrm{I} / \mathrm{R}$ vehicle group $(\mathrm{P}<0.05)$.

Time-course changes in the MBP during MIRI procedure. Fig. 2A details the time-course changes in MBP throughout the MIRI procedure. The recording of MBP commenced $10 \mathrm{~min}$ 
Table I. Effect of tacrolimus on myocardial I/R-induced arrhythmias.

\begin{tabular}{|c|c|c|c|c|c|c|}
\hline \multirow[b]{2}{*}{ Group } & \multirow[b]{2}{*}{$\mathrm{n}$} & \multicolumn{2}{|c|}{ Ventricular tachycardia } & \multicolumn{2}{|c|}{ Ventricular fibrillation } & \multirow[b]{2}{*}{ Mortality } \\
\hline & & Incidence & Duration (sec) & Incidence & Duration (sec) & \\
\hline \multicolumn{7}{|l|}{ Sham } \\
\hline Vehicle & 8 & 0 & 0 & 0 & 0 & 0 \\
\hline Tacrolimus & 8 & 0 & 0 & 0 & 0 & 0 \\
\hline \multicolumn{7}{|l|}{$\mathrm{I} / \mathrm{R}$} \\
\hline Vehicle & 12 & $92 \%$ & $28.5 \pm 6.5$ & $58 \%$ & $35.7 \pm 7.8$ & $33 \%$ \\
\hline Tacrolimus & 12 & $75 \%$ & $10.4 \pm 4.3^{\mathrm{a}}$ & $25 \%{ }^{\mathrm{a}}$ & $15.1 \pm 4.9^{\mathrm{a}}$ & $0 \%{ }^{\mathrm{a}}$ \\
\hline
\end{tabular}

Data are presented as the mean \pm standard error of the mean or as a percentage. ${ }^{a} \mathrm{P}<0.05 \mathrm{vs} . \mathrm{I} / \mathrm{R}+\mathrm{vehicle}$. Sham, rats received sham operation; vehicle, rats received normal saline injection; tacrolimus, rats received $0.1 \mathrm{mg} / \mathrm{kg}$ tacrolimus; $/ \mathrm{R}$, ischemia/reperfusion.

Table II. Effect of tacrolimus on cardiac function.

\begin{tabular}{|c|c|c|c|c|}
\hline \multirow[b]{2}{*}{ Parameter } & \multicolumn{2}{|c|}{ Sham } & \multicolumn{2}{|c|}{$\mathrm{I} / \mathrm{R}$} \\
\hline & Vehicle & Tacrolimus & Vehicle & Tacrolimus \\
\hline Heart rate (bpm) & $214 \pm 10$ & $221 \pm 18$ & $209 \pm 22^{\mathrm{a}}$ & $224 \pm 23$ \\
\hline LVESP (mmHg) & $66 \pm 12$ & $69 \pm 15$ & $36 \pm 6^{\mathrm{a}}$ & $55 \pm 7^{\mathrm{b}}$ \\
\hline LVEDP (mmHg) & $19 \pm 4$ & $17 \pm 5$ & $33 \pm 4^{\mathrm{a}}$ & $20 \pm 5^{\mathrm{b}}$ \\
\hline $\mathrm{dP} / \mathrm{dtmax}(\mathrm{mmHg} / \mathrm{sec})$ & $4,596 \pm 1,325$ & $4,226 \pm 1,635$ & $2,359 \pm 1,014^{\mathrm{a}}$ & $3,452 \pm 1,134^{\mathrm{b}}$ \\
\hline$-\mathrm{dP} / \mathrm{dtmin}(\mathrm{mmHg} / \mathrm{sec})$ & $-4,326 \pm 1,235$ & $-4,259 \pm 1,363$ & $-2,148 \pm 1,127^{\mathrm{a}}$ & $-3,699 \pm 1,364^{b}$ \\
\hline SV/BW ( $\mu 1 /$ gram $)$ & $0.82 \pm 0.45$ & $0.88 \pm 0.39$ & $0.31 \pm 0.15^{\mathrm{a}}$ & $0.75 \pm 0.22^{\mathrm{b}}$ \\
\hline
\end{tabular}

Data are presented as the mean \pm standard error of the mean. ${ }^{\mathrm{a}}<0.05$ vs. sham+vehicle; ${ }^{\mathrm{b}} \mathrm{P}<0.05 \mathrm{vs}$. $\mathrm{I} / \mathrm{R}+\mathrm{vehicle}$. Sham, rats received sham operation; vehicle, rats received normal saline injection; tacrolimus, rats received $0.1 \mathrm{mg} / \mathrm{kg}$ tacrolimus; I/R, ischemia/reperfusion; LVESP, left ventricular end systolic pressure; LVEDP, left ventricular end diastolic pressure; LVEDP, left ventricular end diastolic pressure; $\mathrm{dP} / \mathrm{dtmax}$, maximal rates of rise in left ventricular pressure; - $\mathrm{dP} / \mathrm{dtmin}$, maximal rates of fall in left ventricular pressure; SV/BW, stroke volume/body weight.
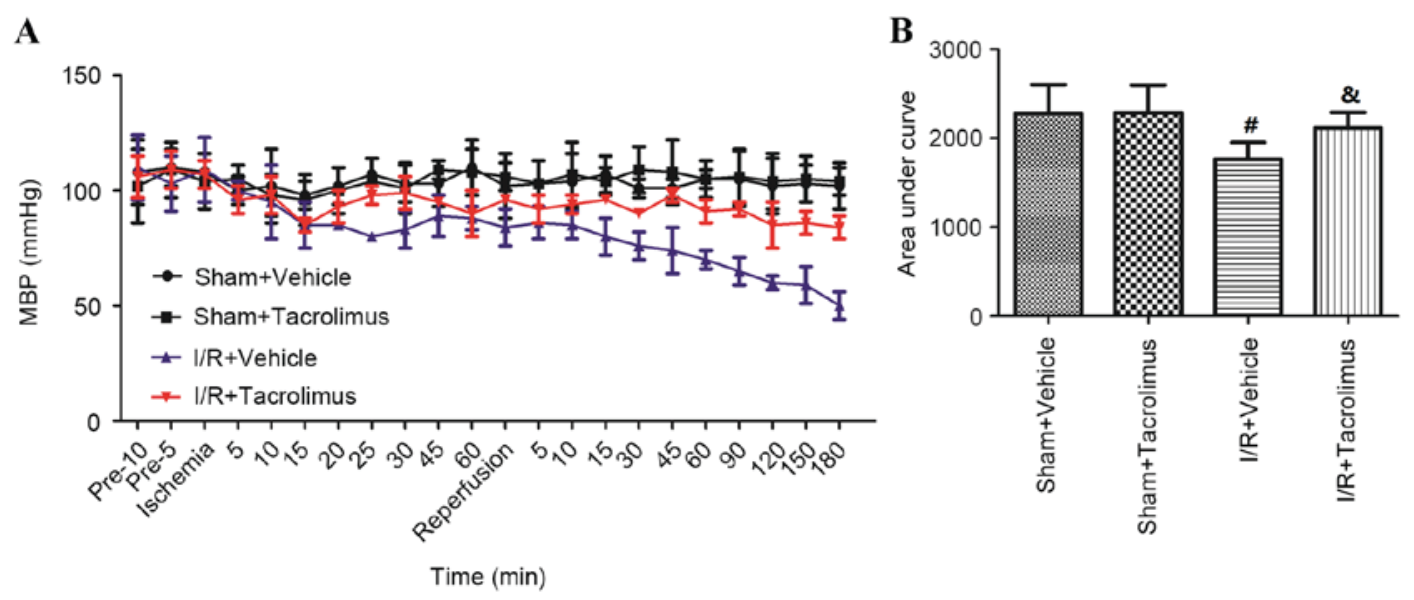

Figure 2. Time-course changes in the MBP during MIRI procedure. (A) Time-course changes in the MBP during MIRI procedure, beginning at 10 min prior to ischemia and ending at $180 \mathrm{~min}$ following reperfusion. (B) Area under the curve calculated from time-course data. Data are presented as the mean \pm standard error of the mean for 12 rats in each group. ${ }^{*} \mathrm{P}<0.05$ vs. sham+vehicle; ${ }^{\&} \mathrm{P}<0.05$ vs. I/R+vehicle. MBP, mean blood pressure; I/R, ischemia/reperfusion; MIRI, myocardial I/R injury.

prior to ischemia and ended at 180 min following reperfusion. No significant change was detected in the sham+vehicle or sham+tacrolimus groups throughout. The MBP in the $\mathrm{I} / \mathrm{R}+$ vehicle and $\mathrm{I} / \mathrm{R}+$ tacrolimus groups began to decrease at 
A

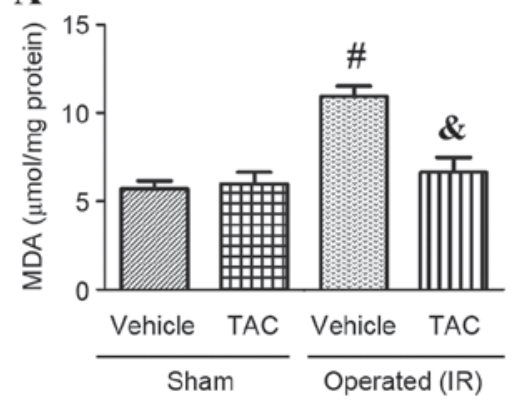

B

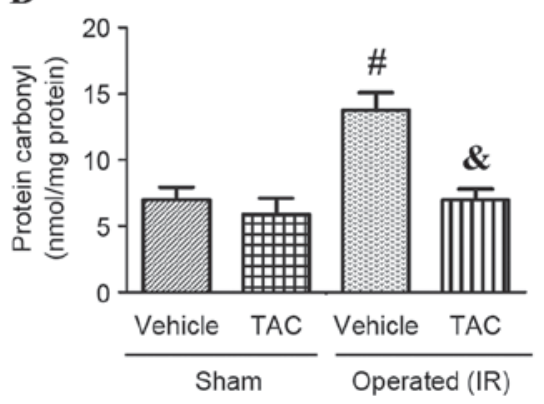

C

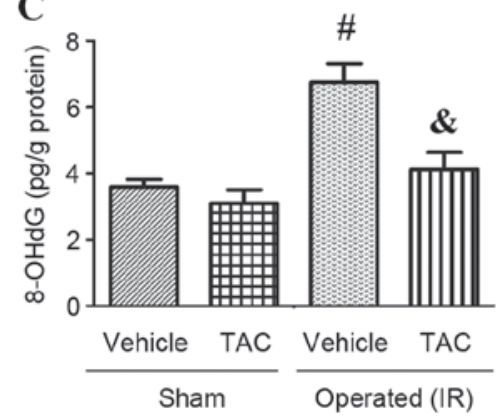

Figure 3. Changes in oxidative products in myocardium. Changes in the levels of (A) MDA, (B) protein carbonyl and (C) 8-OHdGin myocardium following myocardial I/R injury were measured. Data are presented as the mean \pm standard error of the mean for 12 rats in each group. ${ }^{\sharp} \mathrm{P}<0.05$ vs. sham $+\mathrm{vehicle}$; ${ }^{\&} \mathrm{P}<0.05$ vs. I/R+vehicle. MDA, malondialdehyde; 8-OHdG, 8-hydroxy-2-deoxyguanosine; I/R, ischemia/reperfusion; TAC, tacrolimus.

A
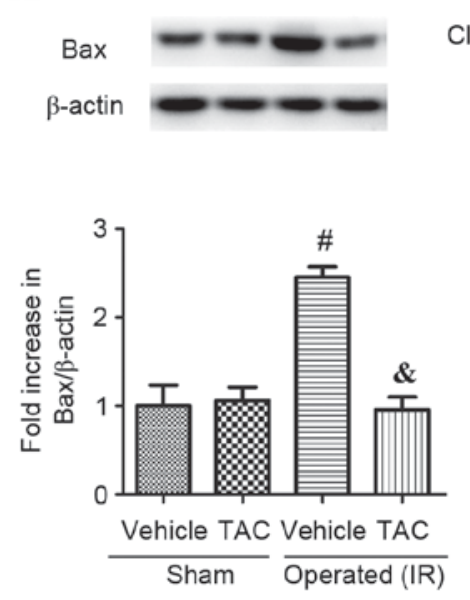

B
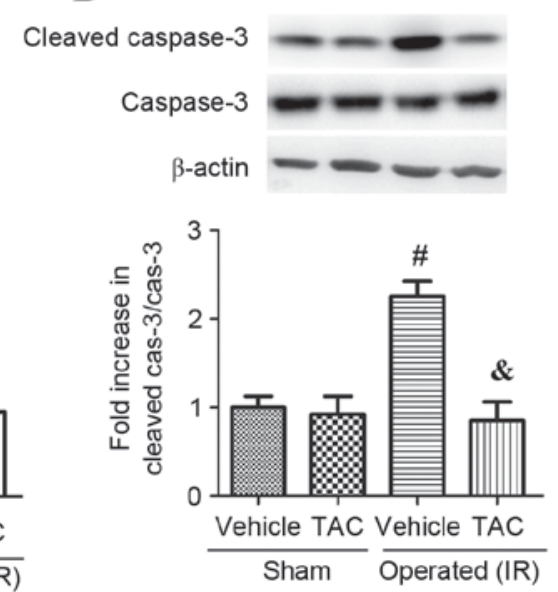

C
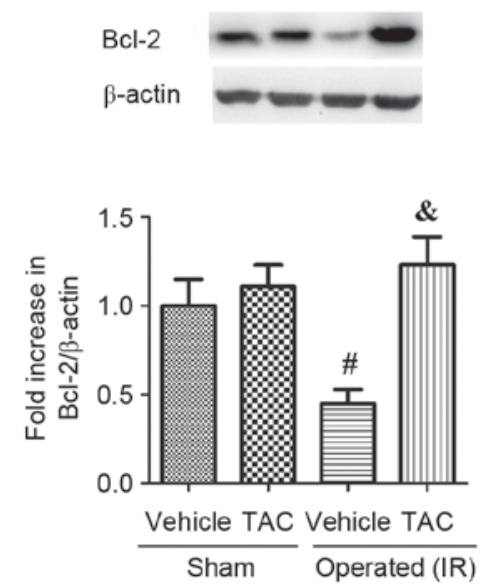

Figure 4. Changes in apoptotic proteins in myocardium induced by myocardial I/R injury and TAC. Changes in the levels of (A) Bax, (B) caspase-3 and (C) Bcl-2 were measured. Data are presented as the mean \pm standard error of the mean for 12 rats in each group. ${ }^{\#} \mathrm{P}<0.05 \mathrm{vs}$. sham $+\mathrm{vehicle}$; ${ }^{\circledR} \mathrm{P}<0.05 \mathrm{vs}$. $\mathrm{I} / \mathrm{R}+\mathrm{vehicle}$. I/R, ischemia/reperfusion; TAC, tacrolimus; Bcl-2, B cell lymphoma 2; Bax, Bcl-2 associated protein $\mathrm{x}$.

15 min following ischemia, but the decrease in the $\mathrm{I} / \mathrm{R}+\mathrm{vehicle}$ was more marked. Fig. 2B demonstrates the area under the curve (AUC) of Fig. 2A. Compared to the sham+vehicle group, the AUC in the I/R+vehicle group was significantly lower $(\mathrm{P}<0.05)$. The AUC in the $\mathrm{I} / \mathrm{R}+$ tacrolimus group was significantly higher than that in the $\mathrm{I} / \mathrm{R}+$ vehicle group $(\mathrm{P}<0.05)$.

Changes in oxidative products $(8-O H d G, M D A$ and protein carbonyl). Fig. 3 depicts changes in the oxidative products (8-OHdG, MDA and protein carbonyl) between groups. MIRI caused significant oxidative stress, as indicated by the significant increase of 8-OHdG, MDA and protein carbonyl levels in the $\mathrm{I} / \mathrm{R}+$ vehicle group vs. the sham+vehicle group $(\mathrm{P}<0.05)$. Tacrolimus reduced oxidative stress, as demonstrated by the significant decreases in these oxidative products compared in the $\mathrm{I} / \mathrm{R}+$ tacrolimus group vs. the $\mathrm{I} / \mathrm{R}+$ vehicle group $(\mathrm{P}<0.05)$.

Changes in apoptotic proteins by MIR and tacrolimus. Fig. 4 details the changes in apoptotic protein level (Bax, caspase-3 and Bcl-2) induced by MIRI and tacrolimus. As shown in Fig. 4A and B, there was a significant increase in Bax and cleaved caspase- 3 levels in the I/R+vehicle group compared with the sham+vehicle group $(\mathrm{P}<0.05)$. Following the treatment of rats in the I/R group with tacrolimus, there was a significant decrease in these levels compared with the I/R+vehicle group $(\mathrm{P}<0.05)$. As shown in Fig. $4 \mathrm{C}, \mathrm{I} / \mathrm{R}+$ vehicle rats exhibited a significant decrease in Bcl-2 levels compared with the sham+vehicle group $(\mathrm{P}<0.05)$. A significant increase in Bcl-2 levels was observed in the $\mathrm{I} / \mathrm{R}+$ tacrolimus group compared with the $\mathrm{I} / \mathrm{R}+$ vehicle group $(\mathrm{P}<0.05)$. As evidenced by the sham groups, treatment with tacrolimus in the absence of I/R did not significantly alter the levels of these apoptotic proteins.

Activation of the PPAR $/$ PI3K/Akt pathway with tacrolimus. Fig. 5 details the activation of PPAR $\gamma / \mathrm{PI} 3 \mathrm{~K} / \mathrm{Akt}$ pathway by the western blot analysis of PPAR $\gamma$ and Akt phosphorylation. As shown in the western blot results, there was no significant difference in protein phosphorylation levels induced between rats exposed to the sham and I/R procedures in the absence of tacrolimus. However, compared with the I/R+vehicle group, the $\mathrm{I} / \mathrm{R}+$ tacrolimus group exhibited significant increases in the phosphorylation levels of both PPAR $\gamma$ and Akt $(\mathrm{P}<0.05)$.

Changes in the cardiac function by inhibitors of PPAR /PI3K/Akt pathway. Table III details changes in cardiac function induced by inhibitors of the PPAR $\gamma / \mathrm{PI} 3 \mathrm{~K} / \mathrm{Akt}$ 
A
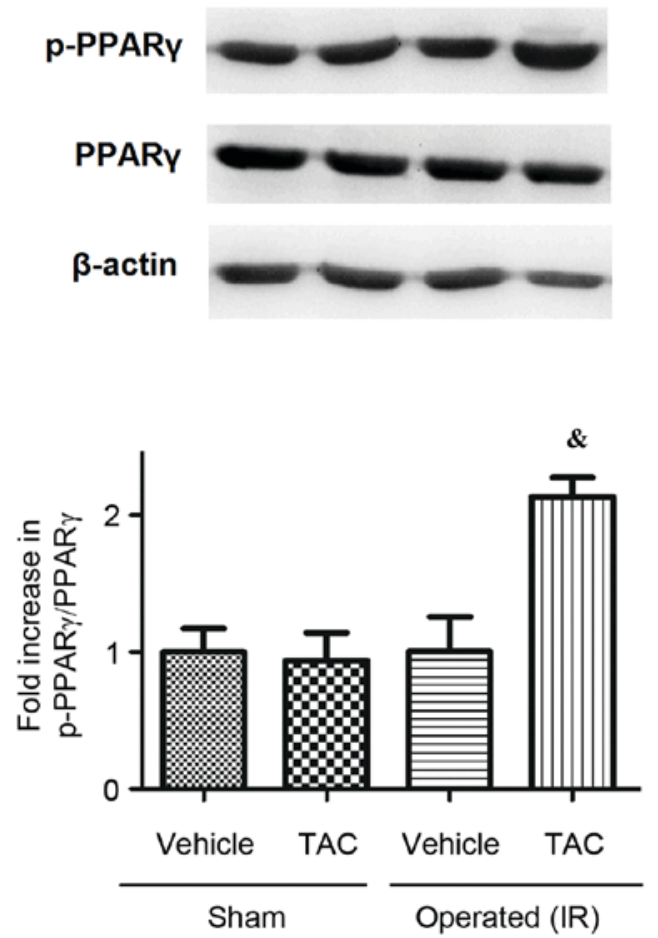

B
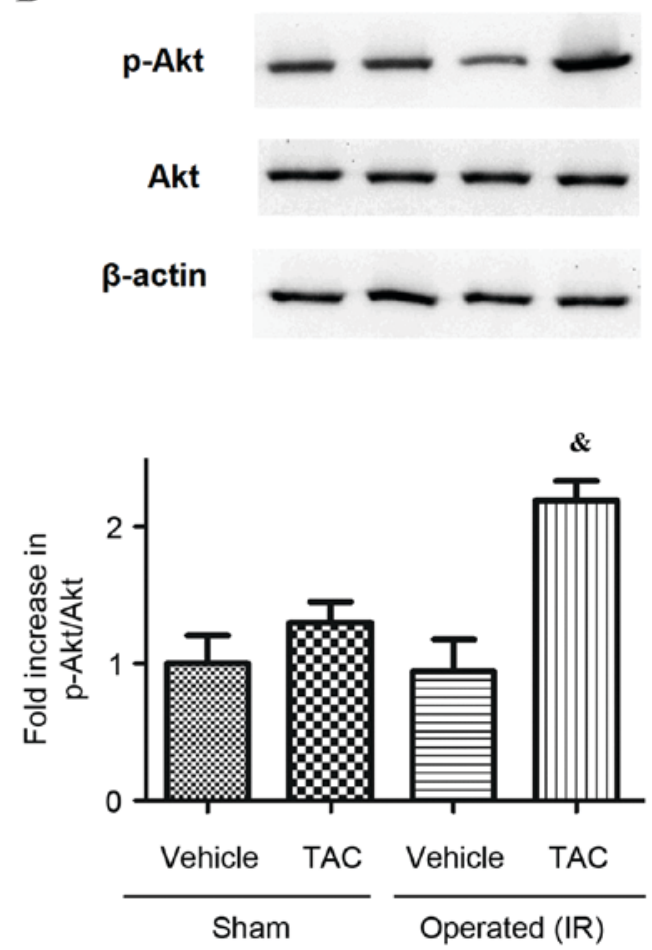

Figure 5. Activation of the PPAR $\gamma / \mathrm{PI} 3 \mathrm{~K} / \mathrm{Akt}$ pathway in myocardium by TAC. Activation of the PPAR $\gamma / \mathrm{PI} 3 \mathrm{~K} / \mathrm{Akt}$ pathway was demonstrated by western blots of (A) PPAR $\gamma$ and (B) Akt phosphorylation. Data are presented as the mean \pm standard error of the mean for 12 rats in each group. \& $<<0.05$ vs. I/R+vehicle. PPAR $\gamma$, peroxisome proliferator-activated receptor $\gamma$; PI3K, phosphoinositide 3-kinase; Akt, protein kinase B; TAC, tacrolimus; I/R, ischemia/reperfusion; p, phosphorylated.

pathway (GW9662, LY294002 or triciribine, respectively). Rats in all the groups were treated with I/R and tacrolimus. Compared with the vehicle group, treatment with LY294001 or triciribine induced significant increases in heart rate, LVEDP and -dP/dtmin, and significant decreases in LVESP, dP/dtmax and SV/BW $(\mathrm{P}<0.05)$. Similar results were observed following GW9662 for all parameters except heart rate, where no significant difference was observed.

Changes induced in PPAR $\mathrm{P}$ or Akt phosphorylation by inhibitors of the PPAR $/$ PI3K/Akt pathway. Fig. 6 details changes of PPAR $\gamma$ or Akt phosphorylation induced by inhibitors of the PPAR $\gamma /$ PI3K/Akt pathway (GW9662, LY294002 and triciribine). As demonstrated in Fig. 6A, GW9662 significantly decreased the phosphorylation of PPAR $\gamma$ compared with the vehicle group $(\mathrm{P}<0.05)$, whereas LY294002 or triciribine did not lead to any significant changes. However, compared with the vehicle group, the phosphorylation of Akt was significantly decreased by treatment with GW9662, LY294002 and triciribine $(\mathrm{P}<0.05)$.

\section{Discussion}

The aim of the present study was to investigate the effect of tacrolimus on arrhythmias, cardiac function, oxidative stress and myocardium apoptosis induced by MIRI, and to elucidate the underlying mechanism. The study was performed in two parts (Fig. 1). In Part 1, rats were divided into four groups: Sham+vehicle, sham+tacrolimus, I/R+vehicle and
$\mathrm{I} / \mathrm{R}+$ tacrolimus, and arrhythmias, cardiac function, MBP, oxidative products, apoptotic proteins, and PPAR $\gamma / \mathrm{PI} 3 \mathrm{~K} / \mathrm{Akt}$ expression and phosphorylation were measured. In Part 2, rats were divided into four groups: I/R+tacrolimus+vehicle, I/R+tacrolimus+GW9662, I/R+tacrolimus+LY294002 and $\mathrm{I} / \mathrm{R}+$ tacrolimus+triciribine, and cardiac functions were examined. The results demonstrated that the MIRI procedure induced arrhythmias and significant impairment of cardiac function, as indicated by changes in the ventricular tachycardia and ventricular fibrillation as well as LVESP, LVEDP, dP/dtmax, -dP/dtmin, SV/BW and MBP. MIRI also caused oxidative stress and apoptosis in cardiomyocytes. Tacrolimus treatment significantly limited the impairments induced by MIR. It alleviated the arrhythmias and significant impairment of cardiac function, and inhibited oxidative stress as well as apoptosis in cardiomyocytes. To investigate the possible mechanism, the role of the PPAR $\gamma / \mathrm{PI} 3 \mathrm{~K} / \mathrm{Akt}$ pathway was investigated. The phosphorylation of PPAR $\gamma$ and Akt were significantly activated by tacrolimus, whereas the inhibitors of PPAR $\gamma /$ PI3K/Akt significantly abolished the effects of tacrolimus. Taken together, these results indicate that tacrolimus may protect rats from MIRI through activation of the PPAR $\gamma /$ PI3K/Akt pathway.

MIRI, which is a result of the recovery of blood flow to the ischemic tissue, may induce electrophysiological perturbation and acute reperfusion arrhythmias in the heart, which may then induce sudden cardiac death $(31,32)$. Electrophysiological perturbation or acute reperfusion arrhythmias may occur either during or following MIRI. Various electrical properties of cardiac tissue may be changed during MIRI, including reduced 
Table III. Effect of PPAR $\gamma$, PI3K and Akt inhibitors on cardiac function.

\begin{tabular}{|c|c|c|c|c|}
\hline \multirow[b]{2}{*}{ Parameter } & \multicolumn{4}{|c|}{$\mathrm{I} / \mathrm{R}+$ tacrolimus } \\
\hline & Vehicle & GW9662 & LY294002 & Triciribine \\
\hline Heart rate (bpm) & $211 \pm 19$ & $213 \pm 17$ & $221 \pm 22^{a}$ & $223 \pm 17^{\mathrm{a}}$ \\
\hline LVESP (mmHg) & $56 \pm 4$ & $33 \pm 5^{\mathrm{a}}$ & $35 \pm 7^{\mathrm{a}}$ & $32 \pm 5^{\mathrm{a}}$ \\
\hline LVEDP (mmHg) & $21 \pm 6$ & $32 \pm 6^{\mathrm{a}}$ & $30 \pm 6^{\mathrm{a}}$ & $36 \pm 5^{\mathrm{a}}$ \\
\hline $\mathrm{dP} / \mathrm{dtmax}(\mathrm{mmHg} / \mathrm{sec})$ & $3,526 \pm 1,125$ & $2,254 \pm 1,123^{\mathrm{a}}$ & $2,175 \pm 1,035^{\mathrm{a}}$ & $2,245 \pm 1,241^{\mathrm{a}}$ \\
\hline -dP/dtmin $(\mathrm{mmHg} / \mathrm{sec})$ & $-3,625 \pm 1,247$ & $-2,243 \pm 1,042^{\mathrm{a}}$ & $-2,011 \pm 1,439^{a}$ & $-2,263 \pm 1,076^{\mathrm{a}}$ \\
\hline $\mathrm{SV} / \mathrm{BW}(\mu 1 / \mathrm{g})$ & $0.77 \pm 0.19$ & $0.35 \pm 0.11^{\mathrm{a}}$ & $0.41 \pm 0.13^{\mathrm{a}}$ & $0.28 \pm 0.10^{\mathrm{a}}$ \\
\hline
\end{tabular}

A
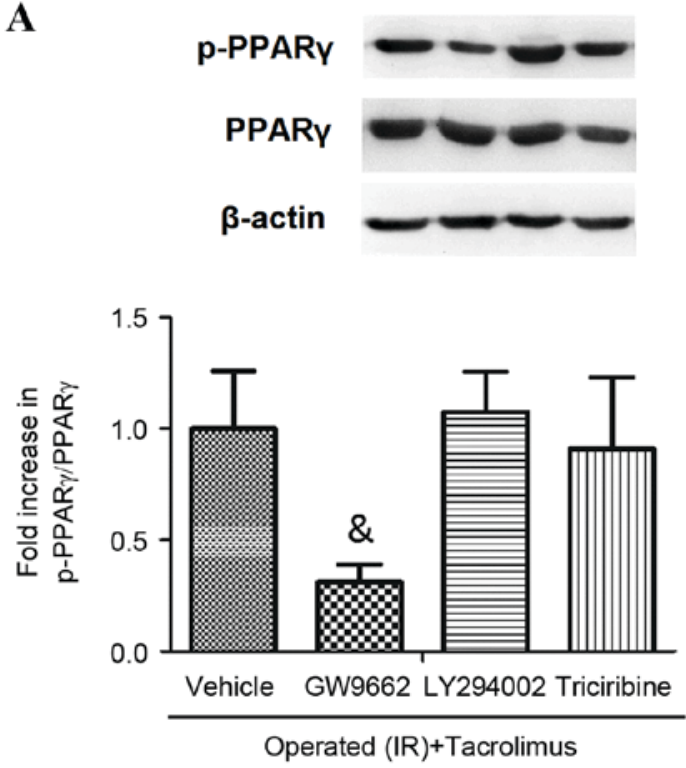

B
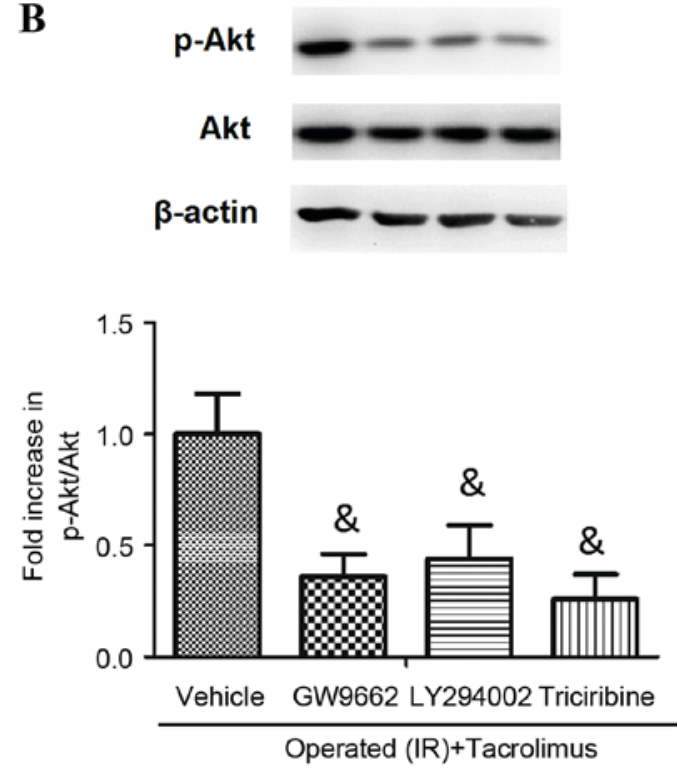

Figure 6. Changes in PPAR $\gamma$ or Akt phosphorylation by inhibitors of the PPAR $\gamma / \mathrm{PI} 3 \mathrm{~K} / \mathrm{Akt}$ pathway. Changes of (A) PPAR $\gamma$ or (B) Akt phosphorylation were demonstrated by the western blot analysis following treatment with GW9662, LY294002 or triciribine. Data are presented as the mean \pm standard error of the mean for 12 rats in each group. ${ }^{\&} \mathrm{P}<0.05$ vs. I/R+vehicle. PPAR $\gamma$, peroxisome proliferator-activated receptor $\gamma$; PI3K, phosphoinositide 3-kinase; Akt, protein kinase $\mathrm{B} ; \mathrm{I} / \mathrm{R}$, ischemia/reperfusion; $\mathrm{p}$, phosphorylated.

resting membrane potential and cellular excitability. It has been reported that the cardiac remodeling following MIRI may alter various ion channels, and decrease cellular excitability and repolarization $(33,34)$. As demonstrated in the present study, MIRI induced a marked increase of incidence and duration of ventricular tachycardia and ventricular fibrillation, indicating that arrhythmia was induced by MIRI. Tacrolimus treatment, however, significantly decreased the duration of ventricular tachycardia, as well as the incidence and duration of ventricular fibrillation, while tacrolimus treatment in the sham group did not cause any significant change in these indicators. To the best of our knowledge, this is the first report to demonstrate that tacrolimus alleviates arrhythmias in an animal model of MIRI.

MIRI may also impair cardiac function, which is demonstrated by hemodynamic parameters and left ventricular function. Hemodynamic parameters were evaluated by heart rate and $\mathrm{MBP}$, whereas left ventricular function was assessed by LVESP, LVEDP, dP/dtmax, -dP/dtmin and SV/BW. MIRI induced a significant decrease in MBP and left ventricular function, but the tacrolimus treatment significantly ameliorated the decline of MBP and left ventricular function induced by MIRI. Tacrolimus treatment alone, however, had no significant effect on hemodynamic parameters or left ventricular function.

Oxidative stress and cardiomyocyte apoptosis are two critical factors in the pathology of MIRI. Over-accumulation of ROS during MIRI overwhelms the anti-oxidative capacity of cells and induces detrimental modifications of lipids, proteins and DNA, thus producing the oxidative products MDA, protein carbonyl and 8-OHdG (35). The present study demonstrated that MIRI significantly increased these oxidative products, which was significantly mitigated by tacrolimus, indicating the anti-oxidative ability of tacrolimus. It has previously been 
demonstrated that the apoptotic process contributes to the loss of cardiomyocytes, myocardial injury and impaired ventricular performance induced by MIRI (36). As tacrolimus significantly ameliorated the cardiac function, the effects of tacrolimus on preventing cardiomyocyte apoptosis were further investigated. The results demonstrated that after rats were treated with tacrolimus, the increase of Bax and cleaved caspase- 3 , and the decrease of Bcl-2 were significantly inhibited. Similar results were obtained in previous studies. Badr et al (19) recently demonstrated that tacrolimus inhibited Bax gene expression and induced Bcl-2 gene expression as an anti-apoptotic gene in a global I/R model. Tacrolimus post-conditioning was also demonstrated to inhibit apoptosis of neural cells and protect the spinal cord from ischemia-reperfusion injury (37). Consistent with these reports, the present study demonstrated the anti-apoptotic property of tacrolimus in MIRI.

Given the previous findings that tacrolimus was able to regulate PPAR $\gamma, \operatorname{PI} 3 K$ and Akt $(21,38,39)$, the role of PPAR $\gamma$, PI3K and Akt in the protection of tacrolimus on MIRI was investigated. PPAR $\gamma$ may regulate the release of vasoactive substances from vascular endothelial cells and the PPAR $\gamma$ activator is able to reverse vascular remodeling, reduce vascular inflammation and improve endothelial function (40). It was previously reported that activation of PPAR $\gamma$ increased endothelial nitric oxide synthase activity and improved the left ventricular remodeling of infracted hearts $(41,42)$. PI3K/Akt serves an essential role in the cardioprotection. The activation of the PI3K/Akt pathway may protect cardiomyocytes from apoptosis following I/R injury $(43,44)$. Li et al $(45)$ recently demonstrated that aqueous extract of cortex dictamni was able to protect cardiomyocytes from hypoxia/reoxygenation-induced oxidative stress and apoptosis via the PI3K/Akt signaling pathway, and Urolithin A was reported to alleviate myocardial I/R injury via the PI3K/Akt pathway (46). In the present study, it was observed that the phosphorylation of PPAR $\gamma$ and Akt was not significantly altered by MIRI procedure, but was significantly increased by tacrolimus. Based on these results, rats were administered with inhibitors of PPAR $\gamma /$ PI3K/Akt (GW9662, LY294002 and triciribine, respectively) and changes in cardiac function were measured. It was demonstrated that co-administration of GW9662, LY294002 or triciribine significantly decreased LVESP, dP/dtmax, and SV/BW, but increased LVEDP and -dP/dtmin, thus abolishing the effects of tacrolimus. Changes in PPAR $\gamma$ or Akt phosphorylation were also measured after rats were administered GW9662, LY294002, triciribine. The results indicated that only GW9662 significantly reduced the phosphorylation of PPAR $\gamma$, whereas GW9662, LY294002 and triciribine significantly reduced the phosphorylation of Akt. These results indicate that PPAR $\gamma$ activation may regulate the PI3K/Akt pathway, but not vice versa. Taken together, these results indicate that tacrolimus suppresses oxidative stress and myocardium apoptosis induced by MIRI via activation of the PPAR $\gamma /$ PI3K/Akt pathway. These results may present a novel signaling mechanism through which tacrolimus protects rats against MIRI in a PPAR $\gamma / \mathrm{PI} 3 \mathrm{~K} / \mathrm{Akt}$ pathway.

The main limitation of the present study was that the role of PPAR $\gamma / \mathrm{PI} 3 \mathrm{~K} / \mathrm{Akt}$ pathway was investigated in the cardioprotective effects of tacrolimus; however other signaling pathways may also be involved. The complete mechanisms regarding the protective effect of tacrolimus against myocardial ischemic/reperfusion injury still require further study.

In summary, the aim of the present study was to examine the protective effect of tacrolimus againstmyocardial I/R injury in an animal model. Although the results demonstrated that tacrolimus significantly alleviated arrhythmia and impairment of cardiac function, and inhibited the oxidative stress and apoptosis in cardiomyocytes, whether tacrolimus is protective in clinical settings requires further investigation.

\section{References}

1. Verheugt FW: Antithrombotic therapy to reduce ischemic events in acute coronary syndromes patients undergoing percutaneous coronary intervention. Interv Cardiol Clin 6: 131-140, 2017.

2. Helft G, Georges JL, Mouranche X, Loyeau A, Spaulding C, Caussin C, Benamer $\mathrm{H}$, Garot P, Livarek B, Teiger E, et al: Outcomes of primary percutaneous coronary interventions in nonagenarians with acute myocardial infarction. Int J Cardiol 192: 24-29, 2015.

3. Yellon DM and Hausenloy DJ: Myocardial reperfusion injury. $\mathrm{N}$ Engl J Med 357: 1121-1135, 2007.

4. Hausenloy DJ and Yellon DM: New directions for protecting the heart against ischaemia-reperfusion injury: Targeting the reperfusion injury salvage kinase (RISK)-pathway. Cardiovasc Res 61: 448-460, 2004.

5. Monassier JP: Reperfusion injury in acute myocardial infarction. From bench to cath lab. Part I: Basic considerations. Arch Cardiovasc Dis 101: 491-500, 2008.

6. Park JL and Lucchesi BR: Mechanisms of myocardial reperfusion injury. Ann Thorac Surg 68: 1905-1912, 1999.

7. Bozlu M, Eskandari G, Cayan S, Canpolat B, Akbay E and Atik U: The effect of poly (adenosine diphosphate-ribose) polymerase inhibitors on biochemical changes in testicular ischemia-reperfusion injury. J Urol 169: 1870-1873, 2003.

8. Ekhterae D, Hinmon R, Matsuzaki K, Noma M, Zhu W, Xiao RP, Gorman RC and Gorman JH III: Infarction induced myocardial apoptosis and ARC activation. J Surg Res 166: 59-67, 2011.

9. Kleinbongard P, Schulz R and Heusch G: TNFa in myocardial ischemia/reperfusion, remodeling and heart failure. Heart Fail Rev 16: 49-69, 2011.

10. Heusch G, Boengler K and Schulz R: Inhibition of mitochondrial permeability transition pore opening: The Holy Grail of cardioprotection. Basic Res Cardiol 105: 151-154, 2010.

11. Kroemer G and Reed JC: Mitochondrial control of cell death. Nat Med 6: 513-519, 2000.

12. Weiss JN, Korge P, Honda HM and Ping P: Role of the mitochondrial permeability transition in myocardial disease. Circ Res 93: 292-301, 2003.

13. Wallemacq PE and Reding R: FK506 (tacrolimus), a novel immunosuppressant in organ transplantation: Clinical, biomedical, and analytical aspects. Clin Chem 39: 2219-2228, 1993.

14. Kim HC, Hwang EA, Han SY, Park SB, Kim HT and Cho WH: Primary immunosuppression with tacrolimus in kidney transplantation: Three-year follow-up in a single center. Transplant Proc 36: 2082-2083, 2004.

15. Jain A, Reyes J, Kashyap R, Rohal S, Abu-Elmagd K, Starzl T and Fung J: What have we learned about primary liver transplantation under tacrolimus immunosuppression? Long-term follow-up of the first 1000 patients. Ann Surg 230: 441-449, 1999.

16. Thomson AW, Bonham CA and Zeevi A: Mode of action of tacrolimus (FK506): Molecular and cellular mechanisms. Ther Drug Monit 17: 584-591, 1995.

17. Ustundag UV, Sahin S, Ak K, Keskin I and Emekli-Alturfan E: The effects of tacrolimus on the activity and expression of tissue factor in the rat ovary with ischemia-reperfusion induced injury. Reprod Biol 15: 139-145, 2015.

18. Pratschke S, Bilzer M, Grützner U, Angele M, Tufman A, Jauch KW and Schauer RJ: Tacrolimus preconditioning of rat liver allografts impacts glutathione homeostasis and early reperfusion injury. J Surg Res 176: 309-316, 2012.

19. Badr R, Hashemi M, Javadi G, Movafagh A and Mahdian R: Assessment of global ischemic/reperfusion and Tacrolimus administration on CA1 region of hippocampus: Gene expression profiles of BAX and BCL2 genes. Bratisl Lek Listy 117: 358-362, 2016. 
20. Chen K, Li JJ, Li SN, Feng J, Liu T, Wang F, Dai WQ, Xia YJ, Lu J, Zhou YQ and Guo CY: 15-Deoxy- $\Delta 12,14$-prostaglandin J2 alleviates hepatic ischemia-reperfusion injury in mice via inducing antioxidant response and inhibiting apoptosis and autophagy. Acta Pharmacol Sin 38: 672-687, 2017.

21. Stechschulte LA, Hinds TD Jr, Ghanem SS, Shou W, Najjar SM and Sanchez ER: FKBP51 reciprocally regulates GR $\alpha$ and PPAR $\gamma$ activation via the Akt-p38 pathway. Mol Endocrinol 28: 1254-1264, 2014.

22. National Research Council; Division on Earth and Life Studies; Institute for Laboratory Animal Research; Committee for the Update of the Guide for the Care and Use of Laboratory Animals: Guide for the Care and Use of Laboratory Animals. 8th edition. The National Academies Press, Washigton, DC, 2011.

23. Zhou C, Bai J, Jiang C, Ye L, Pan Y and Zhang $\mathrm{H}$ : Geranylgeranylacetone attenuates myocardium ischemic/reperfusion injury through HSP70 and Akt/GSK-3//eNOS pathway. Am J Transl Res 9: 386-395, 2017.

24. Su HH, Chu YC, Liao JM, Wang YH, Jan MS, Lin CW, Wu CY, Tseng CY, Yen JC and Huang SS: Phellinus linteus mycelium alleviates myocardial ischemia-reperfusion injury through autophagic regulation. Front Pharmacol 8: 175, 2017.

25. Curtis MJ, Hancox JC, Farkas A, Wainwright CL, Stables CL, Saint DA, Clements-Jewery H, Lambiase PD, Billman GE, Janse MJ, et al: The Lambeth Conventions (II): Guidelines for the study of animal and human ventricular and supraventricular arrhythmias. Pharmacol Ther 139: 213-248, 2013.

26. Sivasinprasasn S, Tanajak P, Pongkan W, Pratchayasakul W, Chattipakorn SC and Chattipakorn N: DPP-4 inhibitor and estrogen share similar efficacy against cardiac ischemic-reperfusion injury in obese-insulin resistant and estrogen-deprived female rats. Sci Rep 7: 44306, 2017.

27. Weng D, Lu Y, Wei Y, Liu Y and Shen P: The role of ROS in microcystin-LR-induced hepatocyte apoptosis and liver injury in mice. Toxicology 232: 15-23, 2007.

28. Levine RL, Wehr N, Williams JA, Stadtman ER and Shacter E: Determination of carbonyl groups in oxidized proteins. Methods Mol Biol 99: 15-24, 2000

29. Umemura T, Tasaki M, Kijima A, Okamura T, Inoue $T$, Ishii Y, Suzuki Y, Masui N, Nohmi T and Nishikawa A: Possible participation of oxidative stress in causation of cell proliferation and in vivo mutagenicity in kidneys of gpt delta rats treated with potassium bromate. Toxicology 257: 46-52, 2009.

30. Musman J, Pons S, Barau C, Caccia C, Leoni V, Berdeaux A, Ghaleh B and Morin D: Regular treadmill exercise inhibits mitochondrial accumulation of cholesterol and oxysterols during myocardial ischemia-reperfusion in wild-type and ob/ob mice. Free Radic Biol Med 101: 317-324, 2016.

31. Bernier M, Manning AS and Hearse DJ: Reperfusion arrhythmias: Dose-related protection by anti-free radical interventions. Am J Physiol 256: H1344-H1352, 1989.

32. Yamazaki S, Fujibayashi Y, Rajagopalan RE, Meerbaum S and Corday E: Effects of staged versus sudden reperfusion after acute coronary occlusion in the dog. J Am Coll Cardiol 7: 564-572, 1986.
33. Motloch LJ and Akar FG: Gene therapy to restore electrophysiological function in heart failure. Expert Opin Biol Ther 15: 803-817, 2015.

34. Donahue JK: Gene therapy for ventricular tachyarrhythmias. Gene Ther 19: 600-605, 2012.

35. Neri M, Fineschi V, Di Paolo M, Pomara C, Riezzo I, Turillazzi E and Cerretani D: Cardiac oxidative stress and inflammatory cytokines response after myocardial infarction. Curr Vasc Pharmacol 13: 26-36, 2015.

36. Mughal W, Dhingra R and Kirshenbaum LA: Striking a balance: Autophagy, apoptosis, and necrosis in a normal and failing heart. Curr Hypertens Rep 14: 540-547, 2012.

37. Pan F, Cheng YX, Zhu CL, Tao FH, Li ZH, Tao HY, He B, Yu L, Ji $\mathrm{P}$ and Tang $\mathrm{H}$ : Tacolimus postconditioning alleviates apoptotic cell death in rats after spinal cord ischemia-reperfusion injury via up-regulating protein-serine-threonine kinases phosphorylation. J Huazhong Univ Sci Technolog Med Sci 33: 852-856, 2013.

38. Love S, Mudasir MA, Bhardwaj SC, Singh G and Tasduq SA: Long-term administration of tacrolimus and everolimus prevents high cholesterol-high fructose-induced steatosis in C57BL/6J mice by inhibiting de-novo lipogenesis. Oncotarget 8: 113403-113417, 2017.

39. Jin J, Jin L, Lim SW and Yang CW: Klotho deficiency aggravates tacrolimus-induced renal injury via the phosphatidylinositol 3-kinase-Akt-forkhead box protein O pathway. Am J Nephrol 43: 357-365, 2016.

40. Li H, Lu W, Cai WW, Wang PJ, Zhang N, Yu CP, Wang DL, Liu BC and Sun W: Telmisartan attenuates monocrotaline-induced pulmonary artery endothelial dysfunction through a PPAR gamma-dependent PI3K/Akt/eNOS pathway. Pulm Pharmacol Ther 28: 17-24, 2014.

41. Yuen CY, Wong WT, Tian XY, Wong SL, Lau CW, Yu J, Tomlinson B, Yao X and Huang Y: Telmisartan inhibits vasoconstriction via PPAR $\gamma$-dependent expression and activation of endothelial nitric oxide synthase. Cardiovasc Res 90: 122-129, 2011.

42. Maejima Y, Okada H, Haraguchi G, Onai Y, Kosuge H, Suzuki J and Isobe M: Telmisartan, a unique ARB, improves left ventricular remodeling of infarcted heart by activating PPAR gamma. Lab Invest 91: 932-944, 2011.

43. Mullonkal CJ and Toledo-Pereyra LH: Akt in ischemia and reperfusion. J Invest Surg 20: 195-203, 2007.

44. Matsui T, Tao J, del Monte F, Lee KH, Li L, Picard M, Force TL, Franke TF, Hajjar RJ and Rosenzweig A: Akt activation preserves cardiac function and prevents injury after transient cardiac ischemia in vivo. Circulation 104: 330-335, 2001

45. Li L, Zhou Y, Li Y, Wang L, Sun L, Zhou L, Arai H, Qi Y and $\mathrm{Xu} \mathrm{Y}$ : Aqueous extract of Cortex Dictamni protects H9c2 cardiomyocytes from hypoxia/reoxygenation-induced oxidative stress and apoptosis by PI3K/Akt signaling pathway. Biomed Pharmacother 89: 233-244, 2017.

46. Tang L, Mo Y, Li Y, Zhong Y, He S, Zhang Y, Tang Y, Fu S, Wang $X$ and Chen A: Urolithin A alleviates myocardial ischemia/reperfusion injury via PI3K/Akt pathway. Biochem Biophys Res Commun 486: 774-780, 2017. 